\title{
A Conceptual Framework for Hetero-specific Human Relationships
}

\author{
Anastasia V. Nikolskaya \\ Moscow State University, Moscow, Russia
}

\author{
Daniel S. Mills \\ University of Lincoln, Lincoln, United Kingdom
}

\begin{abstract}
The theme of human perception itself as a part of nature has arisen. The problem of regulating behavior in human and non-human animals is discussed, and it is hypothesized that behavioral individuality is closely linked to evolutionary development.
\end{abstract}

Keywords: human-animal interaction, a functional system of mentally regulating behavior, hetero-specific group, communication, cognition, emotions, needs

\section{Introduction}

The terms "human-animal interaction" and "anthrozoology" are often used in a scientific context to describe the area relating to the study of how humans and other animals affect each other. However, it might be argued that these terms reflect an anthropocentric bias that has the potential to affect our thinking, by contrasting humans with other animals and perhaps inadvertently focusing attention on the "human" aspect of the relationship. Indeed, the impact of humans on other species tends to be considered more by the discipline of "animal welfare".

However, a relationship is the consequence of an interactive process, which demands equal consideration of all partners (or actors, as people will call them) and their behavior for the relationship to be understood. In addition, the principles which underpin the development of relationships should be universal, varying only on the basis of individual limitations or environmental circumstances. In this paper, firstly, it is considered that some examples of how and why anthropocentricism may have affected scientific endeavor in the life sciences, with special reference to the study of hetero-specific relationships, in order to lay a clearer philosophical foundation for the approach that we propose. Finally, the implications of this approach in relation to the study and management of human-companion animal relationships are considered.

Anthropocentrism describes the tendency for human beings to regard themselves as the central and most significant entities in the universe, or the assessment of reality through a biased human perspective. While any species can only perceive the world around it through its own perceptual mechanisms, it is not difficult to recognize that others may perceive things differently. It is perhaps harder to give balanced consideration to other world views, although an important first step is to acknowledge that humans are merely part of the Earth's ecological system. In cultures with a strong Judaeo-Christian-Muslim influence, man is typically seen as the "pinnacle of creation", and these cultures are also the historical home of the scientific method. Given the 
historical importance and influence of religion in education, it is therefore not surprising that some scientific endeavours have taken an anthropocentric perspective. These biases may be quite subtle, but have the potential to affect our thinking about a subject. For example, it might be argued that the functional approach to social behaviour of Hamilton (1932) has overemphasized the significance of dominant-subordinate relationships within society and the importance to the individual of achieving status over others. When such ideas are applied to human/animal relations, it tends to emphasize human domination over the animal, with humans believing that animals should be subordinate and anticipating behavior consistent with this expectation rather than the more familial relationship that almost certainly exists between humans and companion animals. In this respect, it is notable that the definition of a family has traditionally excluded non-human members, although they may exert important influence, particularly in child development (Nikolskya \& Ulianova, 2009). Similarly, the definition of society often fails to appreciate its multispecies nature and the potential significance of this feature in social cohesiveness and functionality. This anthropocentric bias is endemic and even cultural within the life sciences (for example, in the experiments of Harlow, 1966; Woodworths, 1958; Beninger, Kendall, \& Vanderwolf, 1974; Scott, 1965), and in many of the life sciences, it is common practice to refer to "humans" versus "animals" rather than non-human animals, as in the term "human-animal interactions". Within psychology, human test subjects are often referred to as participants and non-human animal participants as subjects, when there is no logical scientific reason for such a qualitative semantic distinction or when both are reality instruments in the laboratory.

The need to recognize humans as merely part of the biotic environment is not a new idea, as is evident from some of the traditional teachings of Hinduism and Buddhism. Indeed, from a historical perspective, humans have a history of considering themselves to be an integral part of nature that is far older than European culture. Nature was often represented as a living being, perhaps due largely to early man's dependence on the natural environment. Thus, there were no significant differences in human and animal behaviour as both were considered to share a natural unity (Krementsov, 2010). Perhaps concern over anthropogenic causes of global warming and its impact on humans have in part fueled a re-evaluation of man's place in nature. The works of Aristotle were the first to argue for a clear distinction between plants, animals, and humans. He argued that "plant souls" were focused on nourishing and reproduction, while the souls of humans and other animals nurtured aspirations and sensation, while only man had a "rational" soul dedicated to thinking and reasoning. Such a distinction between humans and non-human animals is epitomized in the concept of Cartesian dualism, which was challenged to some extent during the age of enlightenment and the founding of organizations like the royal society. However, even today, scientific and popular research, especially in the field of animal cognition, show an anthropocentric bias in the form of sensational headlines referring to "human-like abilities" in non-human animals rather than describing these as shared (as opposed to human-like) abilities (for example, Khamsi, 2007; Savage-Rumbaugh, Shanker, \& Taylor, 2001).

However, such anthropocentric biases in science are being increasingly recognized and rejected in favour of a broader "naturecentric" approach, which places humans and their activity within a broader biological and ecological context (Panov, 2006). It is, therefore, pertinent to critically appraise the framework used for the study of human-other species relationships.

If it is accepted that everyday attitudes towards animals are formed in line with cultural-historical and social-psychological preconditions, then it should also be recognized that the traditional reductionist scientific approach so widely used in the biological sciences may not be entirely appropriate. Cultural and historical 
preconditions have an impact on relations between humans and non-human animals, and we should recognize the social-psychological nature of these relations. Human relationships with other animals are the product of changing conceptions and linked to social constructs peculiar to a given time, culture, and society. Scientific advances in the life sciences, mathematics, and technology have allowed the investigation of more complex interactive systems with emergent properties, which may facilitate a more expansive evaluation of this subject. To do this is necessary to understand the internal structure of the system and the processes involved in communication, as recognized by ethnologists who have dedicated considerable effort to the study of social structures within populations and their communication channels (Kremetnsov, 2010). This paper aims to explore the values of a possible framework which recognizes these factors for the examination of hetero-specific (human) interactions and relationships.

\section{A Functional System of Mentally Regulating Behavior}

What should an individual perceive within its environment to stimulate adaptive behavior? One of the founders of theoretical biology, Jakob von Uexküll (1926), hypothesized in 1909 that in accordance with its unique special features, each living creature perceives and structures the environment to create an "Umwelt", a world of its own matching the inner world of the living creature and the formation plan of a particular creature - meaning an Umwelt matches living creature, for example, its sense organs, somebody orients in the environment with the help of sight, another one-with the help of sense of smell, etc.; somebody is able to run for a long distance and somebody hides somewhere and waits while victim appears near .

Based on the work of Chuprikova (2007), the authors suggest five functional mental domains for consideration within any psychological model, which may limit or otherwise influence the development and maintenance of hetero-specific (or con-specific) interactions, namely:

\section{The Cognitive System}

First of all, a living being should know its environment. Maturana and Varela (1992) believed that knowledge is not a picture of objective reality, but a method of organizing one's own life experience in order to boost the viability of living organisms. According to the ontological tradition of the theory of cognition, something in the environment penetrates the cognizing subject. This means that the environment is reflected inside a cognizing system. And according to the theory of autopoiesis, a living organism cannot obtain knowledge from outside or go beyond the cognitive picture of reality it creates. The development of a living system's structures depends on its structural conjugacy with the world (one example of structural conjugacy is the gravitational force- It is coordinated with the organism's muscles because the organism has to move in order to survive). A living organism is structurally associated with the world also by means of the sensory organs. The organism has a narrow range of sensations. This narrows the range of what can be seen, heard, smelled, and felt. This makes it possible for a living system to avoid being overloaded with external irritants and form the basis of an acquired knowledge. A distinguishing feature of the process of acquiring knowledge is that it requires repetition, i.e., a situation needs to be recognized as a repetition of another, but similar situation. If everything was absolutely new, it would be impossible to learn anything. For a creature to be able to repeat something, recognition is necessary (identification by means of essential characteristics), and the creature must be able to use this identity again despite a changing situation. According to Maturana and Varela (1992), this identification and subsequent generalization can be carried out only by a mental system. Only when some 
irritants in the environment impact the system and there are ready-made specialized patterns of reacting to events and objects in the environment can the system react to irritants, perceive them as information, and adapt its structure accordingly. The system does not enter into contact with the world of its own accord. It receives irritants of different modalities (visual, acoustic, tactile, and olfactory) and creates information from them with the help of its own apparatus. There is no such information in the surrounding world since only its correlates exist there. Environmental irritants supply the system with something important to it at any given moment, which stimulates search or identification processes. A stimulus from the outside world and the system's reaction to it are not necessarily rigidly bound together-They are controlled by the system's expectations. The system must be able to form expectations to sense possibilities. It needs to have types or schemes to regulate such possibilities. The results of this activity may vary from system to system. The selection is an internal process within the system. It is not represented in the environment in a ready-made form.

Thus, an organism should have knowledge about its environment. This knowledge is gained through sensations, perceptions, memories, thinking, and attention processes.

\section{The Motivational Priority System}

According to Chuprikova (2007), an organism should reflect its own needs and requirements. Maturana and Varela (1992) said that any organism has structural conjugacy with the outside world. The greater the conjugation with the surrounding world, the more irritants in the surrounding world the organism perceives. The greater and more diversified the capacity of a living system to receive irritants from the world, the more accurate information about the world the organism's systems create. The system's expectations, according to Maturana and Varela (1992), are matched by the organism's requirements. These requirements become differentiated with the evolution of the psyche and the organism carries on its search activity in the outside world depending on its requirements. In this sense, information exists inside the organism because as soon as the system generates a requirement and a motive for its satisfaction, the organism initiates an activity to satisfy it consistent with the situation in the outside world as the organism perceives it.

\section{The Emotional System}

In Chuprikova's (2007) theory, an organism should reflect for itself the meanings of external events, inner states, and the consequences of its behavior. To solve this problem of survival, an organism has emotional system.

\section{The Communicative System}

Communication occurs only when an entity understands a message that is received. As a matter of fact, they might misunderstand to some extent, but partial understanding must be sufficient to engender continued communication. Understanding is the basic condition of communication. The messenger knows beforehand whether he will be understood. Communication's informative and meaningful contents have images or structures perceived as a meaning. As in Maturana and Varela's (1992) theory, here, too structures are formed on the basis of the necessity of recurrence and recognition of a communicative situation as a recurrence. This means that communication as well as perception and education processes, requires the ability to identify and generalize. The communication system offers standardized and repetitive sounds or gestures that can be used in different contexts. The system can function only under these conditions. Communicative facts are synchronized as a result of certain structural models being handed down from generation to generation. But communication itself is in no way linked to the natural environment. It has no function of perceiving the natural world. "It is 
necessary to transform the outside world in the psyche through perception. Only thereafter does the psyche decide to expend kinetic energy to report something" (Luhmann, 2004). Thus, a certain level of development of mentality, the exchange of results of reflective activity which occurs by means of communications between different individuals, is necessary.

\section{The Integrative System}

The integrative system is a system which integrates and coordinates information from all the systems and makes a decision regarding behavior.

\section{Behavioral Individuality Is Closely Linked to Evolutionary Development}

The authors describe these further below by reference to interactions between humans and domestic animals, which help to explain:

(1) The behaviour and mental states of both interacting subjects;

(2) The characteristics that a human attributes to an animal (based on their reports) and characteristics that an animal may attribute to a human (according to animal behaviour);

(3) The process of interaction and its products;

(4) The dynamics of the hetero-specific group.

To establish psychological relationships with human an animal should possess it own individuality, its behavior must be regulated not only by inborn programmes, but its individual experience and human expectations.

Gibson's ecological approach (1988) to perception also means that the surrounding world presents such properties and relationships to living creatures that they see, sense, and smell as representatives of their particular biological species consistent with the methods and tasks of their life activity. This means that different species will see the surrounding world in a different way. As the psychic (mental) functions of representatives of the same species differentiate and individual differences within the species widen, they will perceive the world (it will be reflected in their psyche) differently and they will display greater individual change in behavior (in accordance with the constructs built in the process of gaining individual experience). Thus, the authors put forward a hypothesis that behavioral individuality is closely linked to the evolutionary development of the brain and perhaps the key to the development of social relationships. And the logical (rather than experiment-based) proof may be the following: As soon as a deficit of inborn programmes appears in evolution, every living being has to use all remaining resources (body and mind) for adapting to the environment. Absolutely identical conditions are impossible in nature, so every time every living being solves the survival problem differently. Certain individual decisions promoting adaptation may be transferred to future generations through training and imitation, and hence, lead to development. Furthermore, different individuals may cooperate for more successful adaptation when their abilities are used in accordance with individual features, but not with inborn programs (like in social insects), hence, individuality may promote the development of social relationships.

In general, fish and amphibians do not appear to have a large integrative center in their brain, and so their reactions to a stimulus are based largely on a relatively simple hierarchical principle, i.e., the behaviour that appears is dependent on the most strongly stimulated behavioral region of the brain structure. However, given that absolutely identical conditions are impossible in nature, individuality may still arise in these species as a 
result of different developmental paths affecting brain development. With reptiles there is greater development of an integrative brain centre, and so they are able to compare different modes of information and make an individual decision in a quickly changing situation. Thus, reptilians undertake a fuller analysis of perceived stimuli, rather than simply react to whichever is the stronger stimulus (Saveliev, 2005).

\section{What Are Necessary Mental Abilities for Hetero-specific Interaction?}

Within the five domains facilitating individual interactions, there is likely the potential to form meaningful interactive social relationships between species. To facilitate a mutually satisfying hetero-specific human-pet relationship, the animal should possess the following abilities:

(1) Communicative system: The communicative system is the ability to assimilate the communicative signals of another and rapidly exchange information with them. The visual, acoustic, and tactile channels are particularly well adapted for this form of communication, since they are inherently associated with the proximity of another and not durable in the environment. But in the case of hetero-specific interactions, both participants should be able to recognize the communicative signals of the other. This is possible only with a high level of mental development;

(2) Cognitive system: The cognitive system is about: (a) The ability to deconstruct different elements of the environment-The more elements an animal perceives, the better it orients itself in the environment. A domestic animal should perceive different humans and artificial objects (for example, a bowl, a lead, a refrigerator, and so on). In the opposite case, relationships with an animal are not interesting for a human. Hence, these subjects and objects should be stored in long-term memory generalizing and making some form of mental abstraction; (b) The ability to generalize and reason abstractly is necessary to solve a new problem (in the terms of deficit of inborn programmes). Without such an ability in an animal (it may be displayed, for example, when a pet solves a problem to receive a treat), any interactions with such animal become boring for a human; (c) The ability to identify itself with a group and simultaneously differentiate itself as a separate individual in space and time-A pet should perceive itself as a member of the human group, and at the same time it should have its own emotions, needs, etc., that not always correspond to human needs and emotions; and (d) The ability to co-ordinate its own individual opportunities with those of others and coordinate its own action with that of others as a result-The lack of this ability creates serious conflicts with group members;

(3) Emotional system: It is about the ability to generate a range of long-term emotional states, including some form of social attachment;

(4) Motivational priority system: This system is an importance for social bonding, group interaction, and care and attention from others;

(5) Integrative system: The ability is to realize behaviour not only in accordance with the species' typical reactions, but also individual characteristics of the animal (previous experience and possibly temperament) and group expectations.

It is important to appreciate that the foundations of the actors within the system are built on more than the biology of the species involved, i.e., each actor is an individual whose personal development has been influenced by their wider cultural and social setting. Each actor within the hetero-specific group contributes to the construction of a common environment in which the actors mutually develop through a process of interaction (Panov, 2006). From this perspective, the hetero-specific group develops a common "language" (Luhmann, 2004) with its own rules for interaction. This is the product of the system and should be 
distinguished from the process, which is the dynamic temporal characteristics of the interactions that form part of the system. However, the product and process interact, with the inherent characteristics of the system and their prevailing manifestation shaping the interaction that occurs, and the process of interaction shaping the characteristics of the actors involved. However, the product cannot be understood in terms of a static output, since it is always in a dynamic state, in the same way, the speed of a car cannot be understood from a single sample of the static physical properties of the car at a specific point in time.

Similarly, the way or function of any interaction can only be understood in the context of the function for both actors rather than one or other of the actors. In this context, each biological species is functionally equipped to use its own methods of interacting, but there must be some overlap between these methods for meaningful interaction to occur. The experience of this overlap will itself lead to modification in the methods of interacting in the future. As a result, the two species gradually acquire a set of hetero-specific interactive elements in the form of transformed con-specific structures, adapted to interspecies relationships. This set of structures assimilated by each actor, will therefore, by definition, not be simply a characteristic of its species. As the set becomes a condition for adequate interaction, it opens opportunities for new stages of development not previously attainable (Ponomarev, 2010). This concept of a hetero-specific group facilitating mutual but distinct development allows us to consider how to define the maturity of a hetero-specific relationship, such as that occurring between an owner and pet.

Communication involves the transfer between subjects of information that has meaning to both. Although the meaning of the signal sent may not be the same as the message received, a fundamental requirement for efficient communication is some form of common understanding. Signals are standard sounds, gestures, etc., which are repeated and used in different contexts. Thus, communication, like perception and learning processes, requires an ability to generalize and discriminate as a necessary condition for its functioning. Each species will use its own specific abilities to communicate within a relevant hetero-specific relationship, but also learn to detect the communicative signals of the hetero-specific. As a result of this process, each actor teaches the other to orient in response to the communicative signals that are given. As a result, the mutual learning of the components of the interactive system is realized and the "language" of the system forms in a very individualized way. Nonetheless, the language of the system begins to control the behaviour of the actors within the system and assists its development as a whole. In accordance with the principles of social psychology, all social actions directed toward the change of another's behaviour can be classified as either negative compulsion or positive persuasion, and this can be applied to non-human animals as well (Szczepanki, 1969). Each actor within a social interaction typically has some choice, and the purpose of the exchange is to influence the decision of others. In the case of an owner-pet relationship, the human usually has most power (at least initially) in the relationship and so aims to influence the pet's choice of reactions to their own behaviour, without necessarily recognizing that the pet's response involves an attempt to influence the human in return, either to support such behaviour or to bring about a change. In order to understand problematic interactions, it needs to discover the precedents for the problematic interactions to understand the structure of the interaction in the system rather than just the product, i.e., the person initiates problem interactions, the owner's opinion about the initiator, the dynamics of the problem interaction, etc..

During hetero-specific communication, humans are generally guided by visual information (poses and mimicry), with less attention often paid to auditory information, which may be perceived as merely nonsensical "noise", although recent work suggests that humans are sensitive to the qualitative features of different types of 
vocalization associated with different affective states in cats and dogs (Horwitz \& Mills, 2009; Overall, 2005). Only exceptionally is any attention consciously given to olfactory communication, despite its importance in other species. By contrast, non-human animals appear to pay great attention to multiple channels of communication, perhaps because of the need to make a fuller appraisal of the message to be received, given their less powerful position within the system. Accordingly, they may divert greater attentional resources to understanding the signals being communicated during interactions with a human. Inconsistent signals together with a high signal load may in themselves have detrimental psychological effects on the animals, especially in the longer term. Thus, non-human animals can be expected to develop according to their study of the process of human-animal interactions. Within this process the means to learn about external referents, such as gestures or the naming of objects, develop and become psychological tools with which the animal can organize its mental processes, which includes the fundamental components of the interactive system, i.e., cognitive, communicative, integrative, emotional, and motivational systems. The individual experience gained by all participants in the hetero-specific group in the process of their interaction leads to individual development and most probably evolutionary development.

\section{Conclusions}

In today's modern stage of human development, the authors attempt to demonstrate that human understanding is part of nature, but does not have mastery over nature. Firstly, this understanding may occur in the process of interaction with domestic animals if humans admit that an animal possesses its own needs, emotions, wishes, and intentions. Under such conditions, all participants in hetero-specific relationships have the potential for mutual development.

The authors put forward a theoretical hypothesis that behavioral individuality is closely linked to evolutionary development and we have attempted to prove this theory.

The authors believe that consulting work with owners who complain about their pets may be structured around emotional, cognitive, communicative, and motivational priority systems of mentality of hetero-specific interaction participants, when an expert explains an owner that its animal has its own emotion, needs, intentions, etc., which are not always consistent with the owner's, and moreover, these needs, emotions, and intentions are non-human. Despite the above mentioned, it is possible to build hetero-specific relationships that promote the development of participants.

\section{References}

Beninger, R. J., Kendall, S. B., \& Vanderwolf, C. H. (1974). The ability of rats to discriminate their own behaviors. Can. J. Psychol., 28, 79-91.

Chuprikova, N. I. (2007). System of concepts of the general psychology and a functional system of mentally regulating behavior. Voprosy Psikhologii, 3, 3-16.

Gibson, J. J. (1988). The ecological approach to visual perception. Boston: Houghton Mifflin; Moscow: Progress.

Hamilton, W. H. (1932). Institution. Encyclopedia of the Social Sciences, 8, 84.

Harlow, H. (1966). Learning to love am. Scient, 54, 3.

Horwitz, D., \& Mills, D. (2009). BSAVA manual of canine and feline behavioural medicine (2nd ed.). BSAVA.

Khamsi, R. (2007). Dogs show human-like learning ability. Current Biology (doi: 10.1016/j.cub.2007.04.026).

Köhler, W. (1958). The present situation in brain physiology. American Psychologist, 13, 150-154.

Krementsov, N. L. (2010). Human and non-human animals: History of behavioural comparisons. Ethology and Animal Psychology, 2.

Luhmann, N. (2004). Einfuhrung in die systemtheorie. Heidelberg. 
Maturana, H., \& Varela, F. (1992). Tree of knowledge. New York.

Nikolskaya, A. V., \& Ulianova, N. A. (2009). Undirected pet-therapy. Moscow: Aquarium-print.

Overall, K. (2005). Clinical behavioral: Medicine for small animals. Philadeiphia: University of Pennsylvania Press.

Panov, V. I. (2006). Introduction to ecological psychology. Moscow: School Technologies.

Panov, V. I. (2010). Development psychology: Ontological and transcendental preconditions. In N. L. Morina, \& V. I. Panov

(Eds.), Modern perceptual psychology (pp. 293-324). Moscow: URAO Psychological Institute.

Ponomarev, Y. A. (2010). Psyche and intuition. Moscow: Aris.

Savage-Rumbaugh, S., Shanker, S., \& Taylor, T. J. (2001). Apes, language and the human mind (p. 256). Oxford: Oxford University Press.

Saveliev, S. V. (2005). Brain origin. Moscow: Vedi.

Scott, F. (1965). Genetics and social behavior of the dog. American Zoologist, 7, 319-329.

Szczepanski, J. (n.d.). Elementary concepts of sociology. Retrieved from http://www.szczepanski.pl/

von Uexküll, J. (1926). Theoretical biology. New York: Harcourt, Brace \& Co..

Woodworth, R. S. (1958). Dynamics of behavior. New York: Holt. 